\title{
The Influence of Facial Femininity on Chinese and White UK Women's Jealousy
}

\author{
Na Lei ${ }^{1} \cdot$ Hongyi Wang ${ }^{2} \cdot$ Chengyang Han ${ }^{1} \cdot$ Lisa M DeBruine ${ }^{1} \cdot$ Benedict C Jones ${ }^{1}$
}

Published online: 11 September 2018

(C) The Author(s) 2018

\begin{abstract}
It is well established that men report greater jealousy when imagining scenarios in which their romantic partner interacts with men displaying masculine physical characteristics. However, few studies have tested for corresponding effects of sexually dimorphic characteristics on women's jealousy or tested for these effects in non-Western samples. Thus, we investigated the effects of experimentally manipulated sexually dimorphic face-shape cues on Chinese and White UK women's jealousy perceptions. Chinese and White UK women both reported greater jealousy when imagining scenarios in which their romantic partner interacted with more feminine women. Both groups of women showed large effects of facial femininity on jealousy perceptions for both Chinese and White UK stimuli. Together, these results suggest that sexually dimorphic facial characteristics influence women's jealousy and that this effect is not unique to women raised in Western cultures.
\end{abstract}

Keywords Face shape $\cdot$ Jealousy $\cdot$ Sexual dimorphism $\cdot$ Mate preferences $\cdot$ Intrasexual competition

\section{Introduction}

Individuals displaying cues of higher mate value are perceived as greater threats to pair bonds (Dijkstra and Buunk 1998, $2001,2002)$ and threats to pair bonds typically trigger jealousy (Buss et al. 2000). Thus, individuals displaying cues of higher mate value would be predicted to trigger greater jealousy (O'Connor and Feinberg 2012). Since sexually dimorphic features are associated with markers of mate quality, such as attractiveness in women and dominance in men (Little et al. 2011), it may be linked with the perception of jealousy.

Consistent with this quality-jealousy hypothesis, men report greater jealousy when imagining scenarios in which their romantic partner interacts with men displaying more masculine face or body shapes (Dijkstra and Buunk 2001; Kruger 2006; Massar and Buunk 2009; O'Connor and Feinberg 2012) or speaking with a more masculine voice (O'Connor and Feinberg 2012). By contrast with this relatively large body

Benedict C Jones

ben.jones@glasgow.ac.uk

1 Institute of Neuroscience \& Psychology, University of Glasgow, Glasgow, UK

2 School of Psychology and Cognitive Science, East China Normal University, Shanghai, China of work on sexually dimorphic characteristics and men's jealousy, only one study has tested for corresponding effects of sexually dimorphic face-shape characteristics on women's jealousy. Consistent with the quality-jealousy hypothesis, O'Connor and Feinberg (2012) found that Canadian women reported greater jealousy to scenarios in which their romantic partner was imagined interacting with relatively feminine women. These results are consistent with other work reporting that women perceive other women with more feminine face and body shapes to present greater intrasexual competition (Fink et al. 2014) and that women wearing makeup that exaggerates feminine facial color patterns elicit greater jealousy (Mileva et al. 2016). Feminine physical characteristics are thought to reflect good physical condition in women, but not men (see O'Connor and Feinberg 2012), which would explain why feminine physical characteristics in men do not elicit jealousy.

The tendency for research on social judgments of faces to focus on Western participants' perceptions of Western faces is a substantial barrier to our understanding of the generalizability of results across cultures (Sutherland et al. 2018; Han et al. 2018). Consequently, the current study investigated the effects of experimentally manipulated sexually dimorphic face-shape cues on Chinese and White UK women's jealousy perceptions. Previous studies exploring the effects of physical cues on jealousy have examined only Western cultures. 


\section{Methods}

\section{Participants}

Twenty-two heterosexual Chinese women (mean age = 23.98 years, $\mathrm{SD}=3.07$ years) and 15 heterosexual White UK women (mean age $=19.03$ years, $\mathrm{SD}=19.03$ years) took part in the study. All participants were students at the University of Glasgow. Chinese participants were all born in China and reported having been in the UK for an average of 148 days $(\mathrm{SD}=50$ days $)$.

\section{Stimuli}

Face stimuli were manufactured using the same computer graphic methods used by O'Connor and Feinberg (2012). Masculinized and feminized versions of 15 young adult Chinese women's faces were manufactured by transforming the shape of each face image by $\pm 50 \%$ of the linear shape differences between Chinese male and Chinese female face prototypes. Similarly, masculinized and feminized versions of 15 young adult White UK women's faces were manufactured by transforming the shape of each face image by $\pm 50 \%$ of the linear shape differences between White UK male and White UK female face prototypes. Male and female prototypes were manufactured by averaging the shape and color from 50 male and 50 female faces of the relevant ethnicity. Standard computer graphic methods developed for this specific task (DeBruine 2018; see also Tiddeman et al. 2001) were used to manufacture face stimuli. Example stimuli are shown in Fig. 1.

\section{Procedure}

Each participant was shown the 30 pairs of female faces (each pair consisting of a feminized and a masculinized version of the same face). Trial order and the side of the screen on which any given image was presented were fully randomized. Following O'Connor and Feinberg (2012), participants were instructed to "Click on the face of the person who would make you more jealous if they were flirting with your romantic partner (if you do not currently have a romantic partner, imagine that you do have one)."

\section{Results}

For each participant, we calculated the proportion of trials on which she chose the feminized face separately for Chinese faces and White UK faces. Data and analysis code are publicly available at https://osf.io/u24s9/.

One-sample $t$ tests comparing the proportion of trials on which women chose the feminized faces with what would be expected by chance alone (i.e., 0.5) showed that Chinese women selected the feminized faces significantly more often than chance when assessing Chinese $(t(21)=6.481, p<0.001$, $M=0.739, \mathrm{SEM}=0.04, d=1.382)$ and White UK $(t(21)=$ $4.88, p<0.001, M=0.706, \mathrm{SEM}=0.04, d=1.041)$ faces. One-sample $t$ tests also showed that White UK women selected the feminized faces significantly more often than chance when assessing Chinese $(t(14)=6.17, p<0.001, M=0.769$, $\mathrm{SEM}=0.04, d=1.593)$ and White UK $(t(14)=7.051$, $p<0.001, M=0.778, \mathrm{SEM}=0.04, d=1.821)$ faces. Overall, 33 of the 37 women in the study chose feminized faces on more than half of the trials.

We also conducted a $2 \times 2$ mixed-design ANOVA, with face ethnicity as a within-subject factor, and participant ethnicity as a between-subject factor. This analysis showed no significant effect of the ethnicity of participants $(F(1,35)=$ $0.95, p=0.34, \eta \mathrm{p} 2=0.03)$ or the ethnicity of faces $(F(1,35)=0.19, p=0.67, \eta \mathrm{p} 2=0.01)$, and no significant

Fig. 1 Feminized (left) and masculinized (right) versions of a Chinese face used in the study

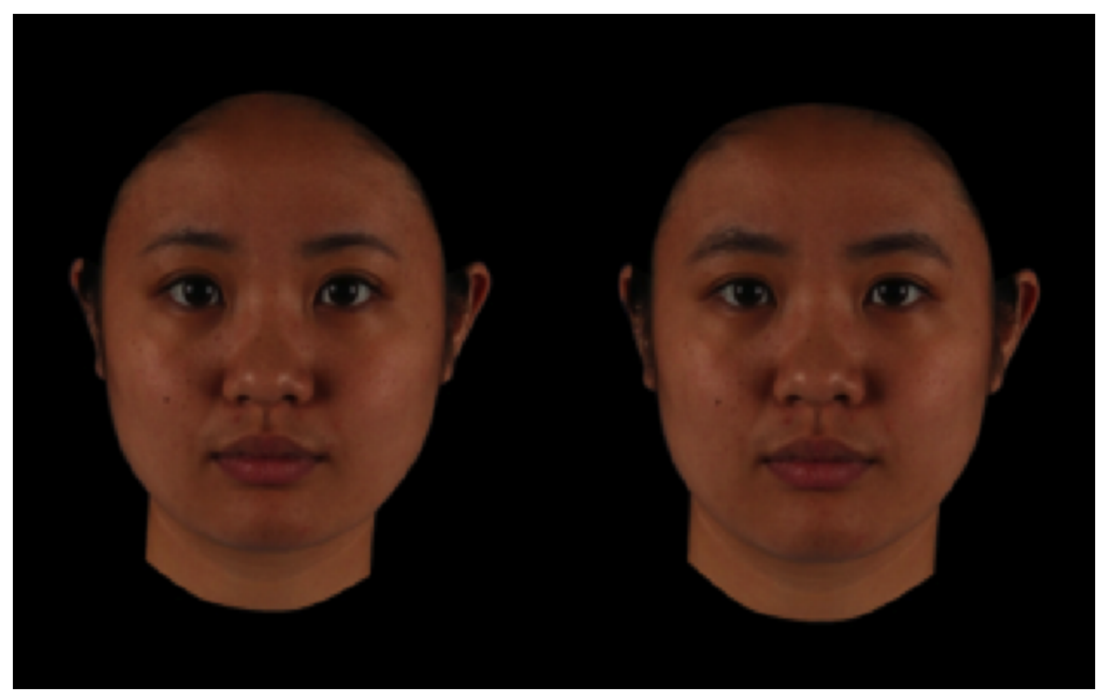


interaction between these two factors $(F(1,35)=0.56, p=$ $0.46, \eta \mathrm{p} 2=0.02$ ).

Finally, we conducted a binomial mixed effects model to simultaneously assess generalizability over stimuli and participants. The dependent variable was the binary preference for the feminized version of each stimulus $(1=$ chose the feminized version, $0=$ chose the masculinized version). The model included face ethnicity as a within-subject fixed factor, participant ethnicity as a between-subject fixed factor, random intercepts for participant and stimulus, and maximally specified random slopes (i.e., face ethnicity within participants). This analysis showed a significant interaction (beta $=1.27, \mathrm{SE}=$ $0.17, Z=7.31, p<0.001,95 \% \mathrm{CI}=[0.93,1.61])$ indicating that participants chose feminized faces more often than masculinized faces. There were no significant effects of the ethnicity of participants (beta $=-0.29, \mathrm{SE}=0.3, Z=-0.97, p=$ $0.334,95 \% \mathrm{CI}=[-0.89,0.3])$ or the ethnicity of faces (beta $=$ $0.02, \mathrm{SE}=0.24, Z=0.08, p=0.94,95 \% \mathrm{CI}=[-0.45,0.49])$, and no significant interaction between these two factors (beta $=0.25, \mathrm{SE}=0.33, Z=0.76, p=0.446,95 \% \mathrm{CI}=[-0.4$, $0.91])$.

\section{Discussion}

We investigated the effects of sexually dimorphic facial characteristics on Chinese and White UK women's jealousy. Both Chinese and White UK participants reported greater jealousy when imagining scenarios in which their romantic partner interacted with more feminine women. Moreover, both Chinese and White UK participants showed this effect when presented with Chinese and White UK faces. Our results for White UK women then replicate O'Connor and Feinberg's (2012) findings for Canadian women's jealousy. Our results for Chinese women demonstrate that the effect of sexually dimorphic facial characteristics on women's jealousy is not unique to women who were raised in Western cultures and also occurs for women raised in an Eastern culture.

Although our analyses demonstrated that both Chinese and White UK women reported greater jealousy when imagining scenarios in which their romantic partner interacted with more feminine Chinese and White UK women, there may be subtle cultural differences in the magnitude of these effects that we did not detect in our study. Further research exploring this issue and also investigating how triggers of jealousy might change with experience of other cultures is a potentially important topic for future research. Similarly, exploring the developmental trajectory of jealousy perceptions to identify when sexually dimorphic physical characteristics in samesex individuals trigger jealousy would provide further insight into the factors that shape jealousy perceptions.

There are limitations to the current study that should be acknowledged. First, the forced choice method we used shows that feminine shape characteristics influence jealousy when faces vary only on that dimension, but cannot show whether these effects are strong enough to influence jealousy when faces vary on other dimensions that also influence jealousy (e.g., makeup). Second, our sample size is relatively small. It would be useful for future work to address these issues.

In conclusion, we show strong effects of sexually dimorphic face shape on jealousy in women. That these strong effects were evident in both Western women (replicating O'Connor and Feinberg 2012) and Chinese women indicates that the effect of sexually dimorphic facial characteristics on women's jealousy is not unique to women who were raised in Western cultures. Strong effects of sexually dimorphic face shape on jealousy in both Chinese and White UK women are also consistent with the strong effects sexually dimorphic characteristics have been shown to have on women's facial attractiveness in both Western and Eastern cultures (Perrett et al. 1998).

\section{Compliance with Ethical Standards}

Conflict of Interest The authors declare that they have no conflict of interest.

Open Access This article is distributed under the terms of the Creative Commons Attribution 4.0 International License (http:// creativecommons.org/licenses/by/4.0/), which permits unrestricted use, distribution, and reproduction in any medium, provided you give appropriate credit to the original author(s) and the source, provide a link to the Creative Commons license, and indicate if changes were made.

\section{References}

Buss, D. M., Shackelford, T. K., Choe, J., Buunk, B. P., \& Dijkstra, P. (2000). Distress about mating rivals. Personal Relationships, 7, 235-243.

DeBruine, L. M. (2018). WebMorph (Version v0.0.0.9001). Zenodo. https://doi.org/10.5281/zenodo.1162670

Dijkstra, P., \& Buunk, A. P. (1998). Jealousy as a function of rival characteristics: an evolutionary perspective. Personality and Social Psychology Bulletin, 24, 1158-1166.

Dijkstra, P., \& Buunk, B. P. (2001). Sex differences in the jealousyevoking nature of a rival's body build. Evolution and Human Behavior, 22, 335-341.

Dijkstra, P., \& Buunk, B. P. (2002). Sex differences in the jealousyevoking effect of rival characteristics. European Journal of Social Psychology, 32, 829-852.

Fink, B., Klappauf, D., Brewer, G., \& Shackelford, T. K. (2014). Female physical characteristics and intra-sexual competition in women. Personality and Individual Differences, 58, 138-141.

Han, C., Wang, H., Hahn, A. C., Fisher, C., Kandrik, M., Fasolt, V., Morrison, D. K., Lee, A., Holzleitner, I. J., DeBruine, L. M., \& Jones, B. C. (2018). Cultural differences in preferences for facial coloration. Evolution and Human Behavior, 39, 154-15959.

Kruger, D. J. (2006). Male facial masculinity influences attributions of personality and reproductive strategy. Personal Relationships, 13, $451-463$. 
Little, A. C., Jones, B. C., \& DeBruine, L. M. (2011). Facial attractiveness: Evolutionary based research. Philosophical Transactions of the Royal Society B: Biological Sciences, 366, 1638-1659.

Massar, K., \& Buunk, A. P. (2009). Rivals in the mind's eye: jealous responses after subliminal exposure to body shapes. Personality \& Individual Differences, 46, 129-134.

Mileva, V. R., Jones, A. L., Russell, R., \& Little, A. C. (2016). Sex differences in the perceived dominance and prestige of women with and without cosmetics. Perception, 45, 1166-1183.

O'Connor, J. J., \& Feinberg, D. R. (2012). The influence of facial masculinity and voice pitch on jealousy and perceptions of intrasexual rivalry. Personality and Individual Differences, 52, 369-373.
Perrett, D. I., Lee, K. J., Penton-Voak, I., Rowland, D., Yoshikawa, S., Burt, D. M., Henzi, S. P., Castles, D. L., \& Akamatsu, S. (1998). Effects of sexual dimorphism on facial attractiveness. Nature, 394, 884-887.

Sutherland, C. A., Liu, X., Zhang, L., Chu, Y., Oldmeadow, J. A., \& Young, A. W. (2018). Facial first impressions across culture: datadriven modeling of Chinese and British perceivers' unconstrained facial impressions. Personality and Social Psychology Bulletin, 44, $521-537$.

Tiddeman, B., Burt, D. M., \& Perrett, D. I. (2001). Computer graphics in facial perception research. IEEE Computer Graphics and Applications, 21, 42-50. 